\title{
Rotator cuff tear with joint stiffness: a review of current treatment and rehabilitation
}

\author{
Hyung-Seok Park, Kyung-Ho Choi, Hyo-Jin Lee, Yang-Soo Kim \\ Department of Orthopedic Surgery, Seoul St. Mary's Hospital, College of Medicine, The Catholic University of Korea, Seoul, Korea
}

\begin{abstract}
Repair of the rotator cuff tear is a joint-tightening procedure that can worsen joint stiffness. This paradoxical phenomenon complicates treatment of rotator cuff tear with joint stiffness. As a result, there is controversy about how and when to treat joint stiffness. As many treatments have been published, this review discusses the latest findings on treatment of rotator cuff tear with joint stiffness.
\end{abstract}

Keywords: Concomitant joint stiffness; Rotator cuff tear; Shoulder; Joint capsule release

\section{INTRODUCTION}

Rotator cuff tear is often accompanied by shoulder stiffness for various reasons [1,2]. Pain from the cuff lesion followed by joint disuse and secondary muscular weakness can lead to shoulder stiffness [3]. The treatment strategy for rotator cuff with concomitant stiffness can be paradoxical [1]. To promote proper healing of the repaired tendon, secure protection and immobilization after the surgery are crucial; however, constant range of motion (ROM) exercise is needed to prevent stiffness. In addition, repair of the torn cuff can exacerbate stiffness because it is a joint-tightening procedure, and postoperative immobilization is important for healing of the repaired tendon [4]. There is no concrete consensus on managing patients having both rotator cuff tear and shoulder stiffness. This review was performed to comprehensively summarize the latest knowledge on treatment of rotator cuff tear with joint stiffness.

\section{DEFINITION AND ETIOLOGY}

The term "frozen shoulder" was first mentioned by Codman [5] as being difficult to define, treat, and explain. The members of the Upper Extremity Committee of the International Society of Arthroscopy, Knee Surgery and Orthopaedic Sports Medicine recently introduced a consensus on the definition of this pathology [6]. According to these authors, the term "stiff shoulder" is used in all patients with restricted ROM, and etiology can be divided into primary or secondary causes. "Frozen shoulder" should be used exclusively as a term to describe the primary idiopathic stiff shoulder that occurs regardless of trauma or specific shoulder disease. Secondary stiff shoulder is used to describe shoulder stiffness with a cause, such as trauma, surgery, or shoulder disease. In this review, we presume the term "stiff shoulder" as secondary stiff shoulder, as it is combined with rotator cuff tear.

Received: April 9, $2020 \quad$ Accepted: April 21, 2020

Correspondence to: Yang-Soo Kim

Department of Orthopedic Surgery, Seoul St. Mary's Hospital, College of Medicine, The Catholic University of Korea, 222 Banpo-daero, Seocho-gu, Seoul 06591, Korea

Tel: +82-2-2258-6117, Fax: +82-2-535-9834, E-mail: kysoos@catholic.ac.kr, ORCID: https://orcid.org/0000-0003-4267-7880

\section{Financial support: None.}

Conflict of interest: None.

Copyright(C) 2020 Korean Shoulder and Elbow Society. All Rights Reserved.

This is an Open Access article distributed under the terms of the Creative Commons Attribution Non-Commercial License (http://creativecommons.org/licenses/by-nc/4.0/) which permits unrestricted non-commercial use, distribution, and reproduction in any medium, provided the original work is properly cited. 


\section{TREATMENT FOR ROTATOR CUFF TEAR WITH JOINT STIFFNESS}

Traditionally, ROM recovery was achieved before surgical repair of a torn cuff [4]. Some studies have insisted on achieving full ROM recovery before surgical treatment [1,7]. Kang [8] asserted that early operation was not needed because the symptoms of rotator cuff tear may not manifest in patients with rotator cuff tear and concomitant adhesive capsulitis. Therefore, sufficient follow-up should be considered for adhesive capsulitis after restoration of shoulder motion [8].

However, non-surgical treatments for shoulder stiffness including stretching, exercise, and manipulation are known to improve scapulothoracic motion rather than glenohumeral joint motion [9]. Moreover, there is a concern that ROM recovery before surgical treatment of rotator cuff tear may deteriorate the condition of the tear, progress muscle atrophy, or produce fat degeneration [10-12]. In fact, there is a possibility that a repairable cuff tear may progress to an irreparable tear during preoperative rehabilitation [13].

According to Oh et al. [14], moderate preoperative shoulder stiffness did not affect clinical outcome in arthroscopic capsular release with manipulation during rotator cuff repair. In other studies, there was no significant difference between a group that underwent capsular release simultaneous with rotator cuff repair and a group that performed rotator cuff repair after ROM recovery $[15,16]$. In addition, several authors have reported satisfactory results through single-stage treatment for rotator cuff tear and joint stiffness $[2,14,17,18]$. According to Cho and Rhee [2], manipulation was performed concurrent with rotator cuff repair, and all groups showed good results. Although the stiffness patients who performed manipulation took longer to recover ROM than those without stiffness, the final outcomes were similar to those in patients without stiffness [2].

Recently, Kim et al. [3] evaluated, in their prospective comparative study, the outcomes of rotator cuff tear with concomitant shoulder stiffness. Specifically, Kim et al. [3] compared immediate surgery in group I versus delayed surgery after 6 months of nonoperative treatment in group II. The author showed significant improvement in ROM and functional scores in both groups at the last follow-up. Moreover, no statistical differences were found in clinical scores (Fig. 1) and ROM, except for internal rotation at 3 and 6 months postoperatively (Fig. 2). Given the lack of benefit to preoperative physical therapy, the authors recommended early surgical treatment of rotator cuff tear with concomitant stiffness using a simultaneous capsular release method [3].
In the case of manipulation under anesthesia (MUA), complications such as fracture, dislocation, osteochondral fracture, rotator cuff tear, anterior labral detachment, superior labral anterior and posterior (SLAP) tears, and radial nerve injury may occur [4]. MUA is effective in improving forward elevation and abduction but is limited in rotation; further, a prior study suggests that fracture can be caused by torsional force [19]. Chuang et al. [20] mentioned that forward flexion and external rotation were improved in the capsular release group compared to the MUA group. According to some authors, simultaneous arthroscopic capsular release can be performed with repair of the torn cuff and improvement of arthroscopic technique. Arthroscopic capsular release has an advantage of meticulous excision or release of the capsule, but also can be an appropriate method for one-stage treatment of rotator cuff tear with joint stiffness [21,22].

\section{ARTHROSCOPIC CAPSULAR RELEASE}

Arthroscopic capsular release is an effective treatment for refractory shoulder stiffness. However, there have been many debates regarding extent of disease, especially regarding the necessity of posterior capsular release [19]. Many studies have revealed that release of the rotator interval improves range of flexion and external rotation $[18,23]$. Several studies have shown that subscapularis tendon, inferior capsule, or global capsule release improves elevation and internal rotation, as well as external rotation [24,25]. Regarding the results of the posterior capsular release, there are some conflicting outcomes. According to the studies by Ide and Takagi [26] and Nicholson [27], posterior capsular release showed improvement in internal rotation. On the other hand, some clinical studies have reported no actual benefit from additional posterior capsular release. Snow et al. [19] suggested that additional posterior capsular release produced no significant difference compared to anterior capsular release. Chen et al. [28] reported similar results that extended posterior capsular release showed no advantage in function or ROM. According to the level I study by Kim et al. [23] that compared the result of arthroscopic release of the anterior/inferior capsule and additional posterior capsular release, there was no significant differences in ROM and clinical outcomes after at least 12 months of follow-up. In addition, Kim et al. (unpublished data) recently tested this suggestion through histologic study. They evaluated anterior and posterior glenohumeral joint capsule tissues from patients with rotator cuff tear who underwent arthroscopic capsular release for shoulder stiffness along with rotator cuff repair. Patients who underwent arthroscopic rotator cuff repair without stiffness were enrolled as 
Pain VAS

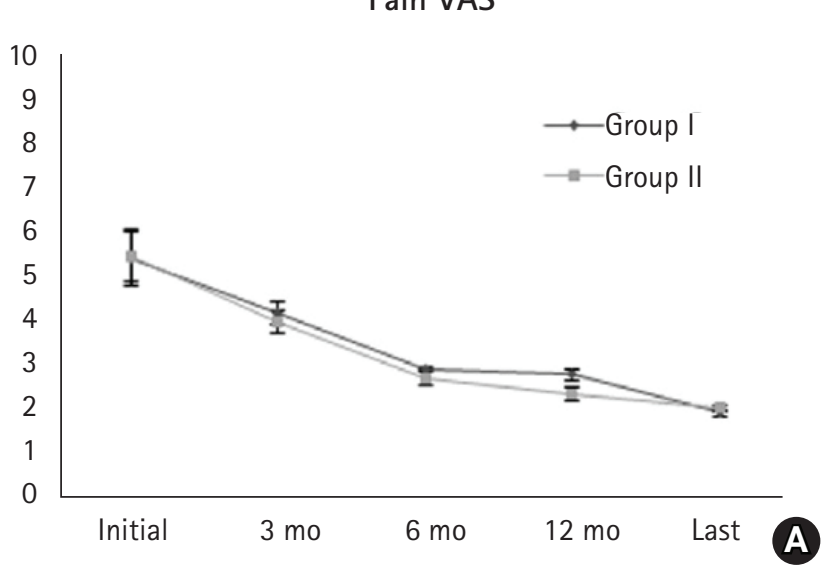

SST

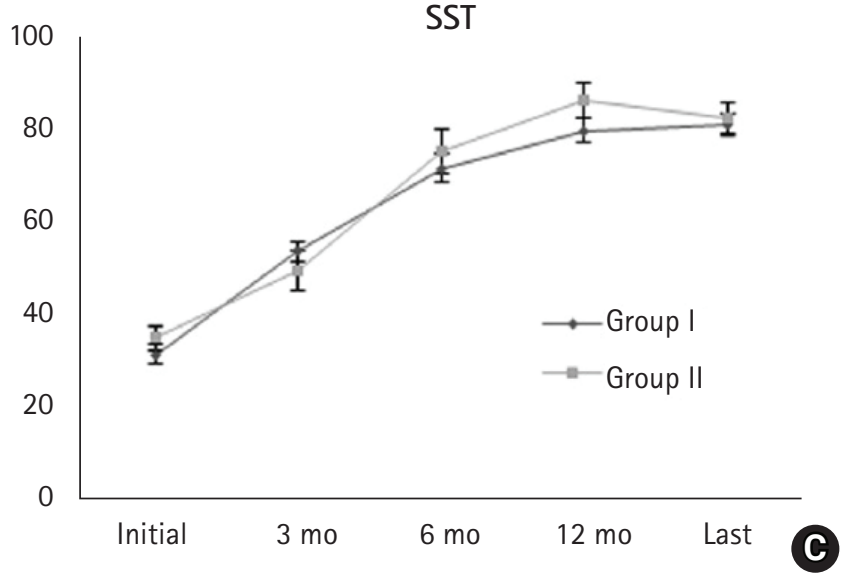

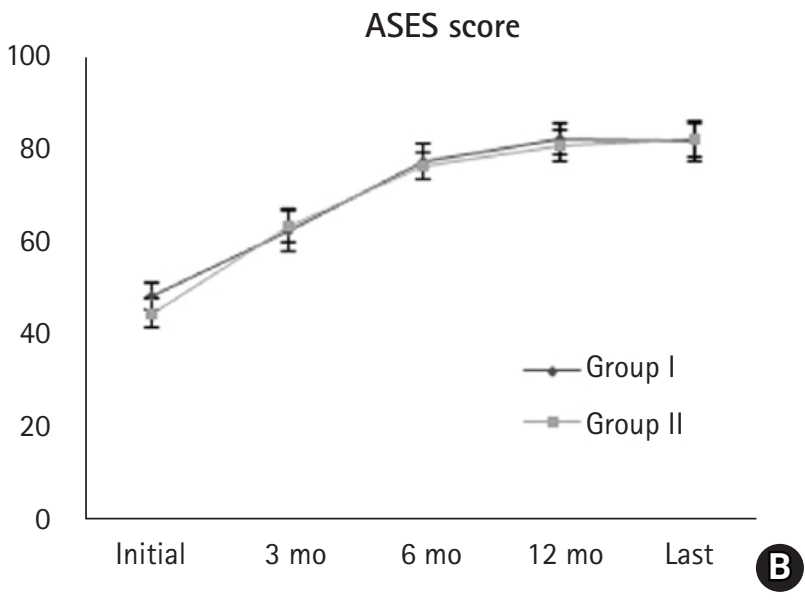

Constant score

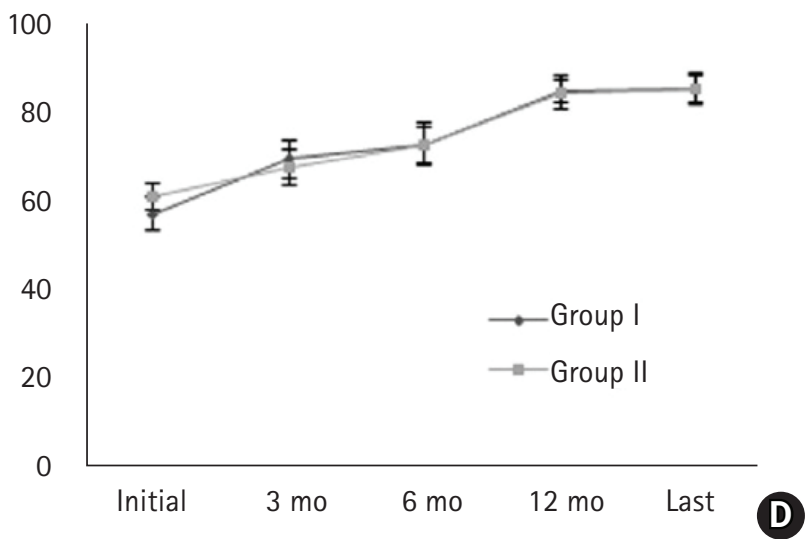

Fig. 1. Functional scores: (A) pain visual analog scale (VAS), (B) American Shoulder and Elbow Surgeons (ASES), (C) Simple Shoulder Test (SST), and (D) Constant. All scores improved after surgery in both groups. No significant differences between groups were seen at any time point.

controls. The gene expression of collagen I and III; fibronectin; extracellular matrix (ECM); basic fibroblast growth factor; transforming growth factor beta; connective tissue growth factor; matrix metalloproteinases (MMPs)-1, MMPs-2, and MMPs-9; tissue inhibitors of metalloproteinase (TIMP)-1 and TIMP-2; intercellular adhesion molecule 1, interleukin-1 and tumor necrotizing factor-alpha were analyzed using real-time reverse transcription polymerase chain reaction. The expression levels of collagen I and III were significantly higher in the anterior capsule compared to those of the posterior capsule and control (Fig. 3). The levels of fibronectin, ECM, MMP-2, and MMP-9 in the anterior capsule were significantly higher than those in the posterior capsule (Fig. 4). Kim et al. (unpublished data) concluded that a more intense fibrogenic process occurs in the anterior capsule compared to posterior and normal capsule tissues, and treatment should be focused on release of the anterior capsule while that of the posterior capsule can be selectively performed.

\section{SURGICAL TECHNIQUE}

The patient is placed either in the lateral decubitus position or in the beach-chair position, depending on surgeon's preference. In cases of lateral decubitus position, the position of the arm varies from $60^{\circ}$ to $70^{\circ}$ of abduction and $15^{\circ}$ to $20^{\circ}$ of forward flexion [15]. Capsular release begins with treatment of the rotator interval and middle glenohumeral ligament via 3.0- $\mathrm{mm} 90^{\circ}$ electrocautery through the anterior portal $[15,19,23]$. Anterior capsular release begins below the biceps origin, preserving the glenoid labrum. Without violating the subscapularis tendon, capsular release is performed to the 7-o'clock (right) or 5-o'clock (left) position involving both the anterior and posterior bands of the inferior glenohumeral ligament $[15,23,28]$. For an easier approach to the inferior and posterior capsules, the working portal is switched to the posterior portal [15]. To avoid axillary nerve damage, capsular release should be performed just off the glenoid rim without violating the glenoid labrum. The closest distance between 
Forward flexion

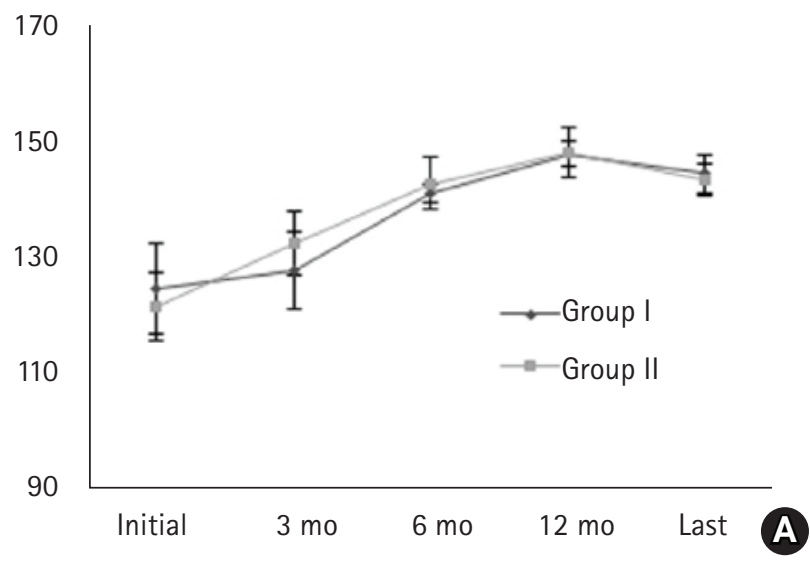

External rotation (at abduction)

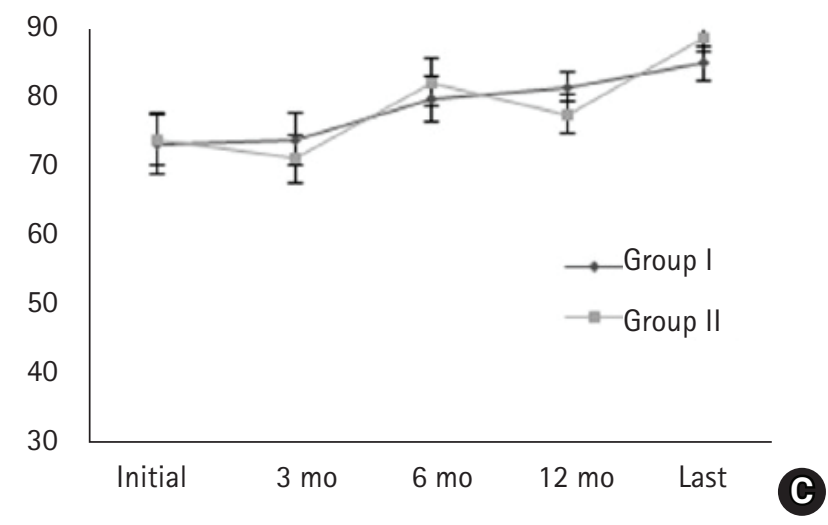

External rotation (at side)

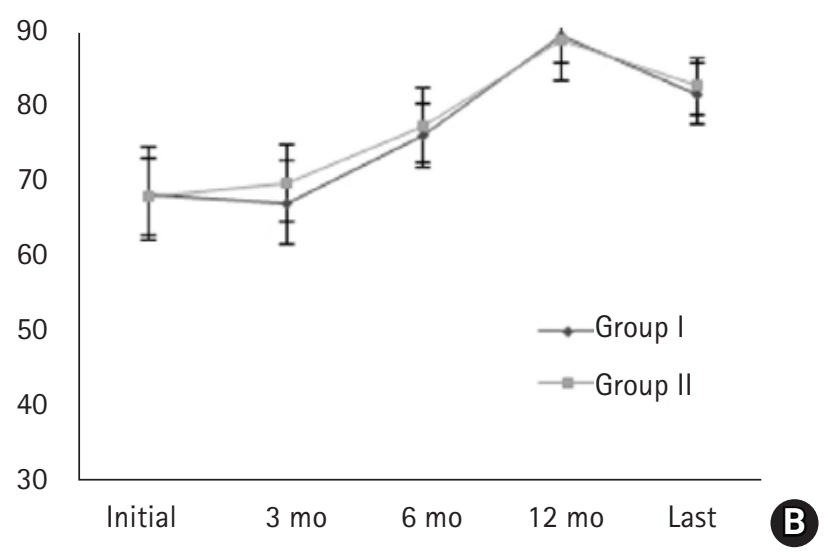

Internal rotation

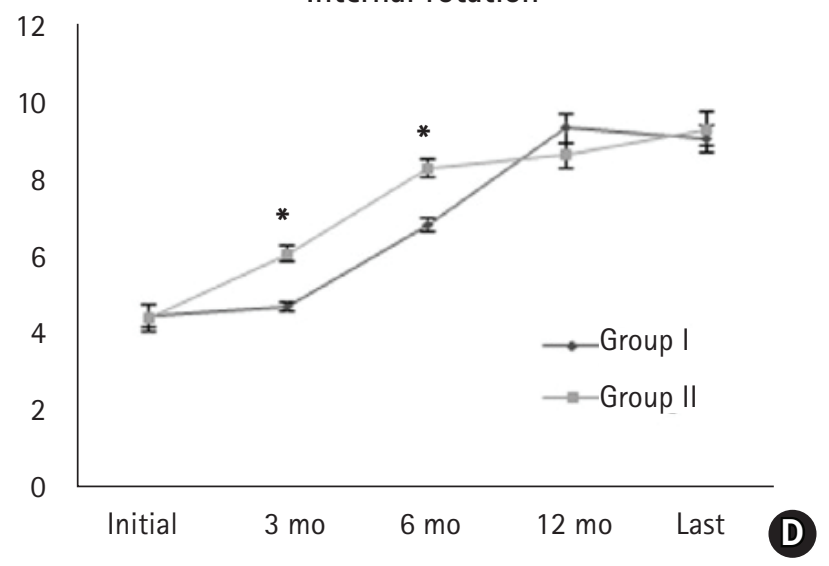

Fig. 2. Passive range of motion (forward flexion [A], external rotation with at side [B] and with abduction [C], and internal rotation [D]) was improved after surgery in both groups. There were no significant differences between the two groups, except for internal rotation (D) at 3 and 6 months. ${ }^{\star} \mathrm{P}<0.05$.

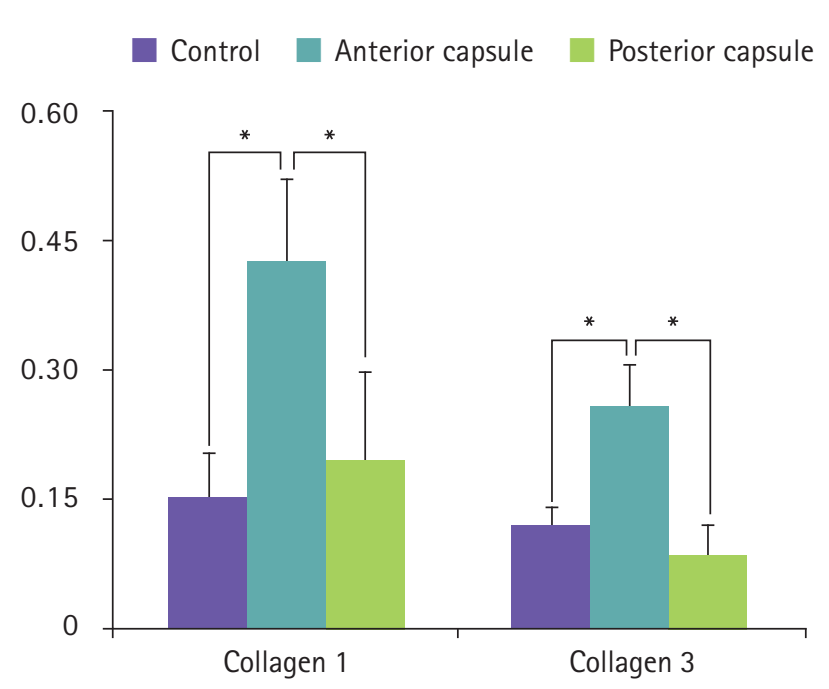

Fig. 3. Gene expression of collagens I and III in the glenohumeral joint capsule. ${ }^{\star} \mathrm{P}<0.05$. the axillary nerve and the glenoid, ranging from 10 to $25 \mathrm{~mm}$, was observed with the arm in the neutral position, and the greatest distance was noted with the arm in an abduction-neutral position [29]. Release of the coracohumeral ligament and the subscapularis is performed with the camera in the lateral portal viewing the anterior portion of the subdeltoid space. The anterior portal is used as a working portal. Using the electrocautery device, the base of the coracoid process is located. The part of the coracohumeral ligament that originates from the coracoid process and extends to the rotator interval is mostly removed during the process of rotator interval tissue removal. However, the coracohumeral ligament extends to the superior part of the subscapularis muscle and covers a broad area of the anterior surface of the subscapularis. For complete release of the coracohumeral ligament, thorough examination and debridement of the anterior and superior portions of the subscapularis are required [15]. 

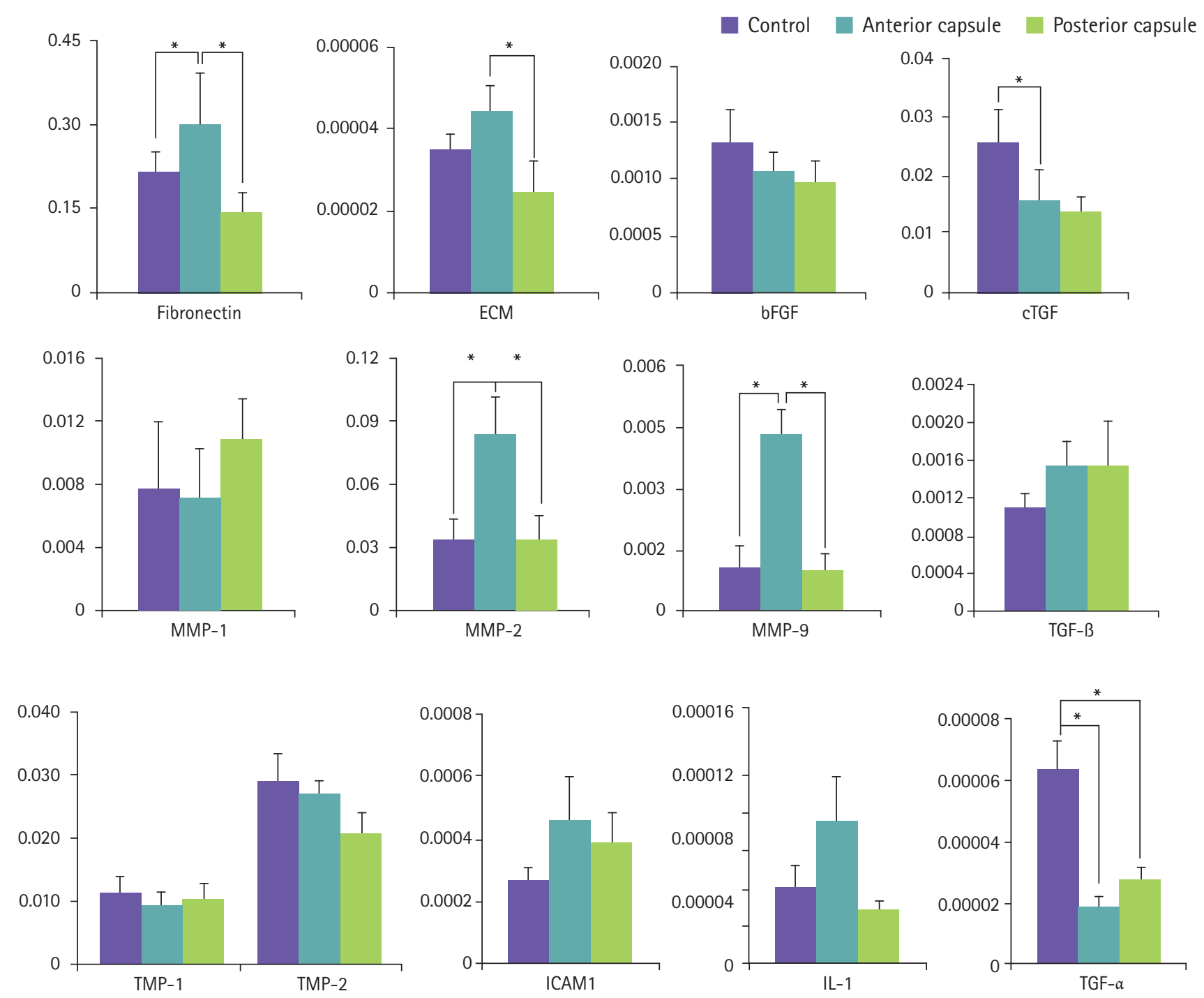

Fig. 4. The gene expression of inflammatory, fibrogenic, and growth factors in the anterior and posterior capsules as well as control tissue. ECM: extracellular matrix, bFGF: basic fibroblast growth factor, cTGF: connective tissue growth factor, MMP: matrix metal, TGF: tumor necrotizing factor, TIMP: tissue inhibitors of metalloproteinase, ICAM: intercellular adhesion molecule, IL: interleukin. ${ }^{\star} \mathrm{P}<0.05$.

\section{POSTOPERATIVE REHABILITATION}

The incidence of shoulder stiffness after rotator cuff repair is reported to be $4.9 \%-32.7 \%[30,31]$. Such joint stiffness may be associated with tear morphology, postoperative immobilization, glenohumeral adhesion, capsular contracture, or underlying predisposing patient comorbidities such as diabetes [6]. To minimize the incidence and duration of postoperative stiffness, various methods were proposed for postoperative rehabilitation. There are many studies about postoperative rehabilitation after rotator cuff tear, though few are comparative studies relating to preoperative rotator cuff tear and concomitant stiffness. In most studies about postoperative rehabilitation, preoperative shoulder stiffness patients were excluded because of restriction of variables. As few studies have investigated postoperative rehabilitation of preoperative shoulder stiffness, the inclusion and exclusion criteria of each study were mentioned herein.

Early passive motion has historically been considered the established protocol to reduce adhesion and stiffness after rotator cuff surgery [32]. Li et al. [33] suggested that continuous passive motion after rotator cuff injury in rabbits promotes basic fibroblast growth factor expression, contributing to tendon recovery by inducing type III collagen synthesis at the tendon-bone interface in the early stages of supraspinatus tendon recovery. According to Cuff and Pupello [34] in their randomized controlled study, early passive motion may help to quickly recover ROM, 
and forward elevation is improved six months after surgery, although patients with accompanying adhesive capsulitis at the time of rotator cuff repair were excluded. However, their study showed no statistical difference between early and delayed rehabilitation 1 year after surgery. Arndt et al. [35] compared immediate passive motion and immobilization after arthroscopic rotator cuff repair in a prospective randomized study. In their study, although preoperative stiffness was not mentioned as an exclusion criterion, the preoperative group had a mean ROM of $174^{\circ}$ and $170^{\circ}$, respectively, and early passive motion showed better functional results with no significant difference in healing of the repaired rotator cuff tendon [35].

Conversely, some recent studies have reported that delayed motion has benefits in clinical and biological outcomes. Sonnabend et al. [36] reported that, at four weeks after surgery, the rotator cuff repair site was still in the early healing phase and remained histologically immature. Gimbel et al. [37] also suggested that delayed motion in the rat model increases the organization of collagen fibers, which subsequently improves tendon to bone healing. Parsons et al. [38] documented favorable outcomes of slower rehabilitation after arthroscopic rotator cuff repair. In their study, all patients underwent full-time sling immobilization without formal therapy for 6 weeks after arthroscopic rotator cuff repair. At 6 to 8 weeks after operation, the patients were allocated into two groups. They categorized patients as "stiff" if they demonstrated forward elevation less than $100^{\circ}$ and external rotation less than $30^{\circ}$ passively; all others were designated as "nonstiff." There was no significant difference in mean ROM, functional scores, or retear rate. They concluded that sling immobilization for 6 weeks after arthroscopic rotator cuff repair did not result in increased long-term stiffness and may improve the rate of tendon healing [38]. Recently, Kim et al. [39] suggested that early passive motion exercise is not mandatory after arthroscopic repair of small to medium-sized full-thickness rotator cuff tears, and postoperative rehabilitation can be modified to ensure patient compliance. In their study, patients were instructed to wear an abduction brace for 4 to 5 weeks after surgery and to start active-assisted shoulder exercise after brace weaning. Group I conducted movement three to four times per day during the abduction brace-wearing period, and group II was allowed no passive motion during the same period. There was no statistical difference in ROM, function score, or tear rate between the early passive motion group and delayed motion group at 1 year follow-up in a prospective randomized study, although patients with preoperative shoulder stiffness were excluded [39]. Therefore, the author recommended that the postoperative rehabilitation protocol be individualized according to patient condition.

\section{CONCLUSION}

Several treatment strategies can be considered when treating rotator cuff tear with joint stiffness. One-stage treatment, namely simultaneous procedures for joint stiffness and repair of the torn cuff, seems to be an effective treatment considering the time required and clinical results. It is also conceivable that arthroscopic capsular release is a useful technique for treating stiffness. When performing arthroscopic capsular release, good results can be obtained even if only anterior and anteroinferior capsules are released. The rehabilitation program can be individualized according to patient compliance.

\section{ORCID}

Hyung-Seok Park https://orcid.org/0000-0003-4781-7539

Kyung-Ho Choi https://orcid.org/0000-0002-3784-1730

Hyo-Jin Lee

https://orcid.org/0000-0002-7708-4754

Yang-Soo Kim

https://orcid.org/0000-0003-4267-7880

\section{REFERENCES}

1. Tauro JC. Stiffness and rotator cuff tears: incidence, arthroscopic findings, and treatment results. Arthroscopy 2006;22:581-6.

2. Cho NS, Rhee YG. Functional outcome of arthroscopic repair with concomitant manipulation in rotator cuff tears with stiff shoulder. Am J Sports Med 2008;36:1323-9.

3. Kim YS, Lee HJ, Park I, Im JH, Park KS, Lee SB. Are delayed operations effective for patients with rotator cuff tears and concomitant stiffness? An analysis of immediate versus delayed surgery on outcomes. Arthroscopy 2015;31:197-204.

4. Weber SC, Abrams JS, Nottage WM. Complications associated with arthroscopic shoulder surgery. Arthroscopy 2002;18 Suppl 1:88-95.

5. Codman EA. Rupture of the supraspinatus tendon and other lesions in and around the subacromial bursa. In: Iannotti JP, Williams GR, eds. The shoulder. 3rd ed. Boston, MA: Thomas Todd Company; 1934. p. 203.

6. Itoi E, Arce G, Bain GI, et al. Shoulder stiffness: current concepts and concerns. Arthroscopy 2016;32:1402-14.

7. Andersen NH, Sojbjerg JO, Johannsen HV, Sneppen O. Frozen shoulder: arthroscopy and manipulation under general anesthesia and early passive motion. J Shoulder Elbow Surg 1998;7: 218-22.

8. Kang SW. Do we have to perform surgical treatment simultaneously for rotator cuff tear in patients with adhesive capsulitis. Korean J Sports Med 2019;37:11-6. 
9. Loew M, Heichel TO, Lehner B. Intraarticular lesions in primary frozen shoulder after manipulation under general anesthesia. J Shoulder Elbow Surg 2005;14:16-21.

10. Gladstone JN, Bishop JY, Lo IK, Flatow EL. Fatty infiltration and atrophy of the rotator cuff do not improve after rotator cuff repair and correlate with poor functional outcome. Am J Sports Med 2007;35:719-28.

11. Goutallier D, Postel JM, Gleyze P, Leguilloux P, Van Driessche S. Influence of cuff muscle fatty degeneration on anatomic and functional outcomes after simple suture of full-thickness tears. J Shoulder Elbow Surg 2003;12:550-4.

12. Yamaguchi K, Tetro AM, Blam O, Evanoff BA, Teefey SA, Middleton WD. Natural history of asymptomatic rotator cuff tears: a longitudinal analysis of asymptomatic tears detected sonographically. J Shoulder Elbow Surg 2001;10:199-203.

13. Gerber C, Fuchs B, Hodler J. The results of repair of massive tears of the rotator cuff. J Bone Joint Surg Am 2000;82:505-15.

14. Oh JH, Kim SH, Lee HK, Jo KH, Bin SW, Gong HS. Moderate preoperative shoulder stiffness does not alter the clinical outcome of rotator cuff repair with arthroscopic release and manipulation. Arthroscopy 2008;24:983-91.

15. Kim YS, Lee HJ. Essential surgical technique for arthroscopic capsular release in the treatment of shoulder stiffness. Version 2. JBJS Essent Surg Tech 2015;5:e14.

16. Oh JH, Kim SH, Ji HM, Jo KH, Bin SW, Gong HS. Prognostic factors affecting anatomic outcome of rotator cuff repair and correlation with functional outcome. Arthroscopy 2009;25:309.

17. Ho WP, Huang CH, Chiu CC, Lee CH, Chen CH, Leu TH, et al. One-stage arthroscopic repair of rotator cuff tears with shoulder stiffness. Arthroscopy 2013;29:1283-91.

18. McGrath JP, Lam PH, Tan MT, Murrell GA. The effect of concomitant glenohumeral joint capsule release during rotator cuff repair--a comparative study. J Shoulder Elbow Surg 2016; 25:714-22.

19. Snow M, Boutros I, Funk L. Posterior arthroscopic capsular release in frozen shoulder. Arthroscopy 2009;25:19-23.

20. Chuang TY, Ho WP, Chen CH, Lee CH, Liau JJ, Huang CH. Arthroscopic treatment of rotator cuff tears with shoulder stiffness: a comparison of functional outcomes with and without capsular release. Am J Sports Med 2012;40:2121-7.

21. Holloway GB, Schenk T, Williams GR, Ramsey ML, Iannotti JP. Arthroscopic capsular release for the treatment of refractory postoperative or post-fracture shoulder stiffness. J Bone Joint Surg Am 2001;83:1682-7.

22. Segmuller HE, Taylor DE, Hogan CS, Saies AD, Hayes MG. Arthroscopic treatment of adhesive capsulitis. J Shoulder Elbow
Surg 1995;4:403-8

23. Kim YS, Lee HJ, Park IJ. Clinical outcomes do not support arthroscopic posterior capsular release in addition to anterior release for shoulder stiffness: a randomized controlled study. Am J Sports Med 2014;42:1143-9.

24. Massoud SN, Pearse EO, Levy O, Copeland SA. Operative management of the frozen shoulder in patients with diabetes. J Shoulder Elbow Surg 2002;11:609-13.

25. Pearsall AW 4th, Holovacs TF, Speer KP. The intra-articular component of the subscapularis tendon: anatomic and histological correlation in reference to surgical release in patients with frozen-shoulder syndrome. Arthroscopy 2000;16:236-42.

26. Ide J, Takagi K. Early and long-term results of arthroscopic treatment for shoulder stiffness. J Shoulder Elbow Surg 2004; 13:174-9.

27. Nicholson GP. Arthroscopic capsular release for stiff shoulders: effect of etiology on outcomes. Arthroscopy 2003;19:40-9.

28. Chen J, Chen S, Li Y, Hua Y, Li H. Is the extended release of the inferior glenohumeral ligament necessary for frozen shoulder. Arthroscopy 2010;26:529-35.

29. Yoo JC, Kim JH, Ahn JH, Lee SH. Arthroscopic perspective of the axillary nerve in relation to the glenoid and arm position: a cadaveric study. Arthroscopy 2007;23:1271-7.

30. Brislin KJ, Field LD, Savoie FH 3rd. Complications after arthroscopic rotator cuff repair. Arthroscopy 2007;23:124-8.

31. Huberty DP, Schoolfield JD, Brady PC, Vadala AP, Arrigoni P, Burkhart SS. Incidence and treatment of postoperative stiffness following arthroscopic rotator cuff repair. Arthroscopy 2009; 25:880-90.

32. Cofield RH. Rotator cuff disease of the shoulder. J Bone Joint Surg Am 1985;67:974-9.

33. Li S, Min SX, Zhang H, Fu GJ, Wang PC, Jin AM. Effect of continuous passive motion on basic fibroblast growth factor expression during tendon-bone repair after surgical repair of acute rupture of the supraspinatus tendon in rabbits. Nan Fang Yi Ke Da Xue Bao 2010;30:1020-3.

34. Cuff DJ, Pupello DR. Prospective randomized study of arthroscopic rotator cuff repair using an early versus delayed postoperative physical therapy protocol. J Shoulder Elbow Surg 2012;21:1450-5.

35. Arndt J, Clavert P, Mielcarek P, et al. Immediate passive motion versus immobilization after endoscopic supraspinatus tendon repair: a prospective randomized study. Orthop Traumatol Surg Res 2012;98(6 Suppl):S131-8.

36. Sonnabend DH, Howlett CR, Young AA. Histological evaluation of repair of the rotator cuff in a primate model. J Bone Joint Surg Br 2010;92:586-94. 
37. Gimbel JA, Van Kleunen JP, Williams GR, Thomopoulos S, Soslowsky LJ. Long durations of immobilization in the rat result in enhanced mechanical properties of the healing supraspinatus tendon insertion site. J Biomech Eng 2007;129:400-4.

38. Parsons BO, Gruson KI, Chen DD, Harrison AK, Gladstone J, Flatow EL. Does slower rehabilitation after arthroscopic rotator cuff repair lead to long-term stiffness. J Shoulder Elbow Surg 2010;19:1034-9.

39. Kim YS, Chung SW, Kim JY, Ok JH, Park I, Oh JH. Is early passive motion exercise necessary after arthroscopic rotator cuff repair. Am J Sports Med 2012;40:815-21. 